Check for updates

Cite this: RSC Adv., 2018, 8, 38987

\section{Preparation of core/shell CdTeahMSN for enhanced tumor vasculature-specific drug delivery}

\author{
Dongzhi Yang, (D) $\dagger^{* a b}$ Na Wang, $\uparrow^{\mathrm{b}}$ Haixia Ji, $\dagger^{\mathrm{b}}$ Shian Sun, ${ }^{\mathrm{c}}$ Jingjing Dong, ${ }^{\mathrm{b}}$ \\ Yuanyuan Zhong, ${ }^{\mathrm{b}}$ Chuntong Qian ${ }^{\mathrm{b}}$ and Huanghuang $\mathrm{Xu}^{\mathrm{b}}$
}

Due to excellent optical properties, CdTe quantum dots (QDs) exhibit great potential in cancer imaging. However, CdTe QDs can be quickly cleared out before reaching the desired location because of their ultra-small size. The structure and optical properties of CdTe QDs are also easily affected by the surrounding solution, which leads to their compromised applications in vivo. Here, CdTe QDs were incorporated into hollow mesoporous silica nanoparticles (hMSN) to form CdTeahMSN nano-platforms. The as-synthesized system maintained the excellent emission properties of CdTe QDs; meanwhile, relatively high drug loading efficiency was also observed for doxorubicin (DOX). With the target for vascular endothelial growth factor (VEGF), the formed CdTeahMSN(DOX)-VEGF Abs showed feasibility of tumor-oriented drug delivery and CdTeahMSN conjugate accumulation. The high accumulation and enhanced targeted drug delivery of CdTeahMSN conjugates in tumor nodules confirmed that CdTeahMSN conjugates can serve as promising candidates for cancer detection and treatment.
Received 29th August 2018 Accepted 8th November 2018

DOI: $10.1039 / \mathrm{c} 8 \mathrm{ra07193d}$

rsc.li/rsc-advances a large cavity in the structure have also attracted significant attention for their ultra-high cargo holding capacity. ${ }^{12,13}$

Blood vessel growth (angiogenesis) is critical for tissue growth, development and remodeling. However, it is also a fundamental step in the transition of tumors from a benign state to a malignant one. When the tumor reaches a certain size (generally 1-2 mm), angiogenesis can occur to supply enough oxygen and essential nutrients. ${ }^{\mathbf{1 4 , 1 5}}$ Tumors induce angiogenesis by secreting various growth factors and proteins. As one of the blood vessel growth factors and a key regulator in the development of tumor, vascular endothelial growth factor (VEGF) and VEGF receptor (VEGFR) pathway in tumor angiogenesis has attracted the interest of researchers in the field of cancer research and therapy. ${ }^{16-18}$ VEGF is mainly concentrated around the tumor vessels, and the response of tumor vessels to VEGF is higher than that of normal vessels, suggesting that VEGF is closely related to tumor angiogenesis. Overexpression of VEGF is associated with the development of multiple tumors and malignant prognosis in colon, breast, prostate and lung cancers. Previous studies have shown that the development of cervical cancer is related to VEGF expression directly. ${ }^{19-21}$ Therefore, the expression specificity of VEGF can result in superior contrast for cancer detection, which makes it an ideal candidate for image-guided drug delivery via nanomaterials.

Targeting of angiogenic markers on tumor vasculature has been accepted as a generally applicable strategy for various nanomaterials regardless of the tumor type. Here, a target recognition and drug delivery system of CdTe@hMSN(DOX)VEGF Abs was established using CdTe QDs as the fluorophore, hMSN as the drug delivery system, and doxorubicin (DOX) as

\footnotetext{
aJiangsu Key Laboratory of New Drug Research and Clinical Pharmacy, Xuzhou Medical University, Xuzhou, Jiangsu 221004, China. E-mail: dongzhiy@xzhmu.edu. cn; Tel: $+86-516-83262138$

${ }^{b}$ Department of Pharmaceutical Analysis, School of Pharmacy, Xuzhou Medical University, Xuzhou, Jiangsu 221004, China

${ }^{c} X u z h o u$ Air Force College, Xuzhou, Jiangsu 221000, China

$\dagger$ These authors contributed equally to this work.
} 
the model drug. The optical and structural properties of the composite nano-system were studied, and targeting recognition was also confirmed. In vitro assays (e.g., flow cytometry and confocal fluorescence microscopy) were carried out to validate the target recognition as well as the cytotoxic effect of the released DOX. Ex vivo experiments (e.g., distribution and histology) were performed on mice bearing HeLa tumors to demonstrate VEGF specificity of VEGF Abs-conjugated CdTe@hMSN. From these experimental data, we inferred that intrinsic CdTe@hMSN(DOX)-VEGF Abs showed great potential to be used for future targeted cancer therapy.

\section{Experimental}

\section{Chemicals and materials}

Hexadecyl trimethyl ammonium chloride (CTAC) and 3-aminopropyltrimethoxysilane (APS) were purchased from Braunwell Chemical Technology Co., Ltd. Tellurium powder, cadmium chloride, glutathione (GSH), sodium borohydride, tetraethyl orthosilicate (TEOS), triethanolamine (TEA), doxorubicin hydrochloride $(\mathrm{DOX} \cdot \mathrm{HCl})$, and sodium hydroxide were purchased from Shanghai Aladdin Biochemical Technology Co., Ltd. Sodium carbonate, sodium chloride, methanol, and anhydrous ethanol were acquired from Sinopharm Group Chemical Reagent Co., Ltd. Fetal bovine serum (FBS) was acquired from Gibco Biochemical Technology Co., Ltd. 3-(4,5-Dimethylthiazol2-yl)-2,5-diphenyltetrazolium bromide (MTT), dimethyl sulfoxide (DMSO) and 6-diamidino-2-phenylindole (DAPI) were purchased from Nanjing KeyGen Biotechnology Inc. Dulbecco's modified Eagle's medium (DMEM), roswell park memorial institute medium (RPMI)-1640, and trypsin were purchased from Vicmed (Xuzhou, China). SCM-PEG-Mal (i.e., succinimidyl carboxymethyl PEG maleimide, MW: $5 \mathrm{kDa}$ ), Traut's reagent, tris(2-carboxyethyl)phosphine (TCEP) reagent were acquired from Thermo Fisher Scientific, Inc. VEGF Abs was acquired from Abcam Biochemical Technology Co., Ltd. Cy3-labeled donkey anti-rat IgG was purchased from Beijing Boao Sen Biotech Co., Ltd. All buffers were prepared using Milliporegrade water. HeLa and L929 cells were provided by Jiangsu Key Laboratory of New Drug Research and Clinical Pharmacy. All reagents were directly used without further purifications following manufacturer's instructions.

\section{Synthesis of CdTe@hMSN nanoparticles}

CdTe QDs were prepared using a previously published method with GSH as the stabilizer, where the molar ratio of Cd : Te : GSH was $2: 1: 3 .^{22,23}$ The CdTe QDs with maximum emission wavelength of $550 \mathrm{~nm}$ were acquired after refluxing the precursor at $100{ }^{\circ} \mathrm{C}$ for $3 \mathrm{~h}$. CdTe QDs were precipitated using isopropanol to obtain the powder. The core/shell CdTe@hMSN were synthesized using a modified Stöber method ${ }^{24}$ and $\mathrm{Na}_{2} \mathrm{CO}_{3}$-etching process. ${ }^{25,26}$ Briefly, the prepared CdTe QDs (5 mg) were first dissolved in $20 \mathrm{~mL}$ of $1: 1$ mixture of methanol and water ( $\mathrm{pH}$ value adjusted with $0.4 \mathrm{~mL}$ ammonia), followed by addition of $0.5 \mathrm{~mL}$ TEOS and allowed to react for $1 \mathrm{~h}$ at room temperature (RT) to form $\mathrm{CdTe} \& \mathrm{SiO}_{2}$. After sequentially washing with ethanol and water, $\mathrm{CdTe} \& \mathrm{SiO}_{2}$ was redispersed in $10 \mathrm{~mL}$ of water. Then, $1 \mathrm{~g}$ CTAC and $100 \mu \mathrm{L}$ TEA (10\%) were thoroughly mixed in $10 \mathrm{~mL}$ water, followed by addition of the prepared $\mathrm{CdTe} \& \mathrm{SiO}_{2}$ solution for reaction at RT for $1.5 \mathrm{~h}$. Without any treatment, $80 \mu \mathrm{L}$ TEOS was added dropwise into the solution to allow the mixture to react at $80^{\circ} \mathrm{C}$ for $1 \mathrm{~h}$ to form $\mathrm{CdTe} \& \mathrm{SiO}_{2} @ \mathrm{SiO}_{2} \mathrm{NPs}$. Using $\mathrm{Na}_{2} \mathrm{CO}_{3}$ as the etching reagent, $318 \mathrm{mg}$ of $\mathrm{Na}_{2} \mathrm{CO}_{3}$ was added to etch the inner $\mathrm{dSiO}_{2}$ at $50^{\circ} \mathrm{C}$ for $30 \mathrm{~min}$. The core/shell CdTe@hMSN powder was obtained after washing with $\mathrm{NaCl}$ methanol (1\%) extraction to remove CTAC. Finally, APS was hydrolyzed for further functionalization with amino-groups to form CdTe@hMSN- $\mathrm{NH}_{2}$. Here, the asprepared CdTe@hMSN was first dispersed in $10 \mathrm{~mL}$ absolute ethanol, followed by addition of $0.5 \mathrm{~mL}$ APS and the reaction was maintained at $86-90^{\circ} \mathrm{C}$ in a water bath for $24 \mathrm{~h}$. Afterwards, the mixture was washed with absolute ethanol three times to remove any residual APS. CdTe@hMSN- $\mathrm{NH}_{2}$ was dissolved in water and amine group concentration was determined using a Kaiser test kit.

\section{CdTe@hMSN functionalization}

VEGF-Abs was mixed with Traut's reagent at a molar ratio of $1: 30$ at $\mathrm{pH} 8.0$ for the incorporation of thiols onto the antibody molecules. After $2 \mathrm{~h}$ of incubation at RT, the resulting VEGFAbs-SH was purified by PD-10 column using phosphatebuffered saline solution (PBS) as the mobile phase. Based on the titration results from Ellman's reagent, we calculated that there were 5 thiol groups per VEGF-Abs on an average under this reaction condition.

CdTe@hMSN- $\mathrm{NH}_{2}$ was reacted with SCM-PEG ${ }_{5 \mathrm{k}}-\mathrm{MAL}$ at $\mathrm{pH}$ 8.5 at a molar ratio of $1: 25$ for $2 \mathrm{~h}$. After removing unreacted SCM-PEG ${ }_{5 \mathrm{k}}$-MAL by purifying with $50 \mathrm{k}$ Amicon filter, CdTe@hMSN-PEG-MAL was obtained. Subsequently, CdTe@hMSN-PEG-MAL was mixed with VEGF-Abs-SH at a molar ratio of $1: 6$ at pH 8.0 in the presence of TCEP to protect thiols from oxidation. After reacting for $12 \mathrm{~h}$ at $4{ }^{\circ} \mathrm{C}$, the final product CdTe@hMSN-VEGF Abs was collected by PD-10 purification.

\section{Material characterization}

The morphology of CdTe@hMSN conjugates was evaluated by G2T12 transmission electron microscopy (TEM) (FEI, USA). Meanwhile, their hydrodynamic size and size distribution as well as $\zeta$-potentials were determined by a 380 ZLS dynamic light scattering (DLS) instrument (Nicomp, USA). The loading amount of CdTe QDs in hMSN and probable cadmium ion release were measured by inductively coupled plasma atomic emission spectroscopy (ICP-AES) (PE, USA). Ultraviolet-visible (UV-Vis) and emission spectra assays were carried out on Hitachi U-3010 and F-4600 fluorescence spectrometers (Hitachi, Japan), respectively.

\section{Cell cytotoxicity assay of CdTe@hMSN conjugates}

Cell cytotoxicity analysis was performed using MTT assay. HeLa and L929 cells were seeded in a 96-well plate at a density of $6 \times$ $10^{3}$ cells per well. After separate incubations with 
CdTe@hMSN-NH ${ }_{2}, \quad$ CdTe@hMSN-PEG, and CdTe@hMSNVEGF Abs for $24 \mathrm{~h}$, the relative viabilities of cell samples were determined by a cell titer following the vendor's protocols. The percentages of viable cells relative to the untreated control were plotted against CdTe@hMSN conjugate concentrations.

\section{Drug loading and releasing measurement}

DOX was used as a model drug to test the drug loading capacity of CdTe@hMSN. DOX·HCl aqueous solution was adjusted to pH 8.0 with TEA, followed by mixing with CdTe@hMSN conjugate (CdTe@hMSN or CdTe@hMSN-VEGF Abs) at a mass ratio of $1: 1$ based on the amount of CdTe@hMSN. The mixture was stirred for $24 \mathrm{~h}$ at RT, and excess DOX was removed by filtration through a $2 \mathrm{kDa}$ filter with repeated rinsing of PBS. The drug release was evaluated at $37{ }^{\circ} \mathrm{C}$ in simulated physiological condition at the $\mathrm{pH}$ values of 5.0, 6.5, and 7.4. CdTe@hMSN(DOX)-VEGF Abs or CdTe@hMSN(DOX) was placed in a dialysis bag with a molecular weight cut-off of $2 \mathrm{kDa}$. The dialysis bag was immersed in the release medium and kept in a shaker (100 rpm) under RT. Samples of $0.2 \mathrm{~mL}$ volume were periodically removed and the same volume of fresh medium was added. The loading and releasing rates of DOX in CdTe@hMSN(DOX)-VEGF Abs and CdTe@hMSN(DOX) were calculated by determining unbound DOX in the washing/ releasing solutions by UV-visible spectrometry. The DOX release studies were performed in triplicate for each sample.

\section{Flow cytometry and fluorescence microscopy}

HeLa and L929 cells were harvested and suspended in PBS buffer (supplemented with $2 \%$ bovine serum albumin) at a concentration of $2 \times 10^{6}$ cells per $\mathrm{ml}$, incubated with CdTe@hMSN and CdTe@hMSN-VEGF Abs $\left(2 \mu \mathrm{g} \mathrm{mL}^{-1}\right.$ based on CdTe@hMSN) for $1 \mathrm{~h}$ at $37^{\circ} \mathrm{C}$, and washed three times with PBS. Afterwards, the cells were resuspended in PBS and analyzed using a MACSQuant Analyzer (Miltenyi Biotec GmbH, Germany). Cellular fluorescence was computed via FlowJo (X.0.7, Tree Star, Ashland, OR). The cells were also examined under an Olympus FV10i (Olympus, Japan) confocal microscope with $200 \times$ magnification to validate the flow cytometry results.

\section{In vivo tumor accumulation}

All animal procedures were performed in accordance with the National Academy of Sciences Guide for the Care and Use of Laboratory Animals of USA ${ }^{27}$ and approved by the Animal Ethics Committee of Xuzhou Medical University. Tumors were established by subcutaneous injection of $2 \times 10^{6}$ of HeLa cells suspended in $100 \mu \mathrm{L}$ of PBS into the female nude mice. The tumor sizes were monitored every other day and the mice were subjected to imaging studies when the tumor diameter reached 5-8 $\mathrm{mm}$. The mice were sacrificed at $2 \mathrm{~h}$ p.i., and the tumor and muscle were removed for ex vivo fluorescence imaging in the Berthold LB983 NightOWL II system (485/600 nm for DOX). To confirm the in vivo tumor accumulation of CdTe@hMSN conjugates, frozen tumors were cut into slices of $6 \mu \mathrm{m}$ thickness. After being fixed with cold acetone for $10 \mathrm{~min}$, tumors were rinsed with cold PBS and blocked with $2 \%$ BSA for $30 \mathrm{~min}$. Subsequently, the tissue slides were stained for endothelial marker CD31 with a rat antimouse CD31 antibody $\left(2 \mu \mathrm{g} \mathrm{mL} \mathrm{m}^{-1}\right)$ for $1 \mathrm{~h}$, followed by Cy3labeled donkey anti-rat IgG $\left(3 \mu \mathrm{g} \mathrm{mL}^{-1}\right)$ for $2 \mathrm{~h}$. The locations of CdTe@hMSN conjugates were visualized using the fluorescence of CdTe QDs (which emitted at $550 \mathrm{~nm}$ under $350 \mathrm{~nm}$ excitation). All fluorescence images were taken with an IX73 digital microscope (Olympus, Japan) with $200 \times$ magnification.

\section{Results and discussion}

\section{Material characterization}

CdTe@hMSN were found to be core/shell nanocrystals with CdTe QDs as the core and hMSN as the shell. Based on TEM measurements in Fig. 1, the individual sizes of CdTe QDs, hMSN, and CdTe@hMSN were about $3.0 \pm 1 \mathrm{~nm}, 90 \pm 10 \mathrm{~nm}$, and $90 \pm 10 \mathrm{~nm}$, respectively. The sizes obtained from DLS curve were larger than that obtained from TEM images, which resulted from the hydration of NPs in aqueous solution. By measuring the total amount of Cd (from CdTe QDs) using ICPAES testing, the loading efficiency of $0.15 \mathrm{mg}$ CdTe QDs $/ \mathrm{mg}$ hMSN was calculated.

CdTe@hMSN was modified with APS hydrolysate to form amine groups on the surface. The amine group concentration determined by the ninhydrin coloration method was about 200 $\mathrm{nmol} \mathrm{mL}{ }^{-1}$. After reacting with SCM-PEG ${ }_{5 \mathrm{k}}$-MAL, it could be linked with an antibody by covalent binding. As shown in Fig. 2A and B, the optical properties such as excitation and emission did not vary after conjugation with the VEGF antibody. Compared with the result for rhodamine 6G (95\%), the fluorescence yield of CdTe@hMSN was $12.3 \%$. As shown in Fig. 2C, two fluorescence emission peaks of CdTe@hMSN and DOX confirmed the successful drug loading in CdTe@hMSN(DOX)VEGF Abs. The DLS results in Fig. 2D also indicated that the size of CdTe@hMSN conjugates increased slightly after surface modification with SCM-PEG ${ }_{5 \mathrm{k}}$-MAL and VEGF Abs from $c a$. $100 \mathrm{~nm}$ (CdTe@hMSN) to $c a .110 \mathrm{~nm}$ (CdTe@hMSN-VEGF Abs). The successful surface engineering was further validated by $\zeta-$ potential measurements, as shown in Fig. 2E, in which a significant change in surface charge was observed after SCM$\mathrm{PEG}_{5 \mathrm{k}}$-Mal coating ( $\zeta$-potential: from $3.49 \pm 0.31 \mathrm{mV}$ to -41.40 $\pm 2.3 \mathrm{mV}$ ). After conjugating with VEGF Abs, the surface charge of CdTe@hMSN-VEGF Abs was $-4.25 \pm 0.21 \mathrm{mV}$, which was similar to that of CdTe@hMSN(DOX)-VEGF Abs. The probable cadmium ion release rate from CdTe@hMSN was measured by detecting the cadmium concentration using ICP-AES. After incubating CdTe@hMSN in different media for 7 days, the percentages of released cadmium were all lower than $1.5 \%$ and were significantly lower than those for naked CdTe QDs. The results indicated that hMSN played an important role in enhancing the stability of CdTe QDs.

\section{DOX loading and release}

Based on the hollow structure, hMSN is definitely suitable for loading of various cargos. The amount of DOX loaded into the CdTe@hMSN conjugates was calculated to be $0.743 \mathrm{mg} \mathrm{mg}^{-1}$ CdTe@hMSN conjugates based on the absorbance 

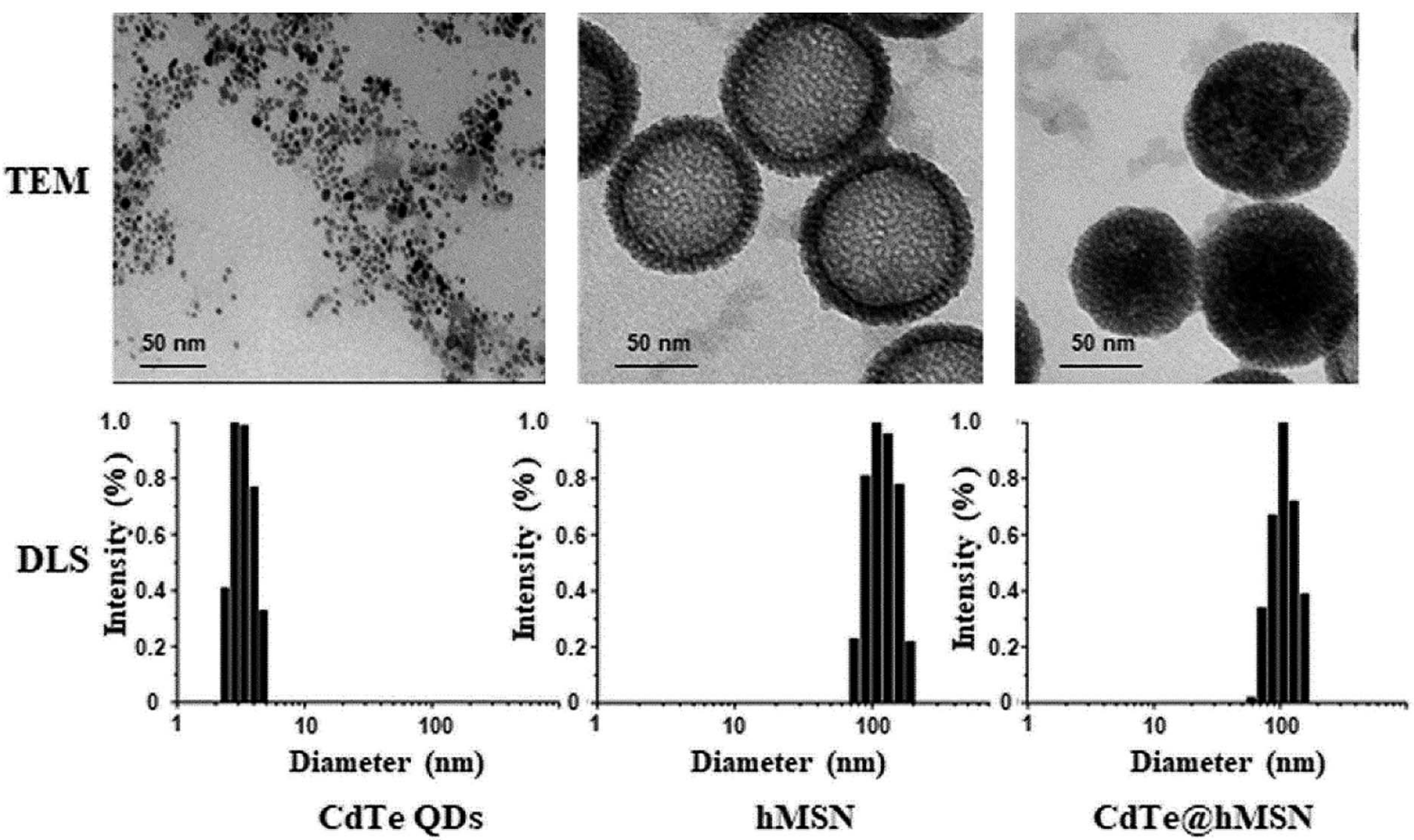

Fig. 1 The morphology of CdTe QDs, hMSN, and CdTeahMSN.

measurement at $490 \mathrm{~nm}$ for DOX. Under simulated physiological condition at the $\mathrm{pH}$ values of 5.0, 6.5 and 7.4 and at $37{ }^{\circ} \mathrm{C}$, the DOX release profile from CdTe@hMSN(DOX) was measured. The results shown in Fig. 3A indicated that the medium $\mathrm{pH}$ had an impact on the release rate of DOX from the CdTe@hMSN conjugates. At pH 7.4, 21.2\% of DOX was released after $72 \mathrm{~h}$, which suggested that loaded DOX within CdTe@hMSN was relatively stable under physiological condition. In contrast, when medium $\mathrm{pH}$ was decreased to 5.0 (mimics endocytic compartments, where the $\mathrm{pH}$ ranges from 4.5 to 6.5 ), the amount of released DOX increased to approximately 62.74\% (0.47 mg DOX/mg CdTe@hMSN). The results
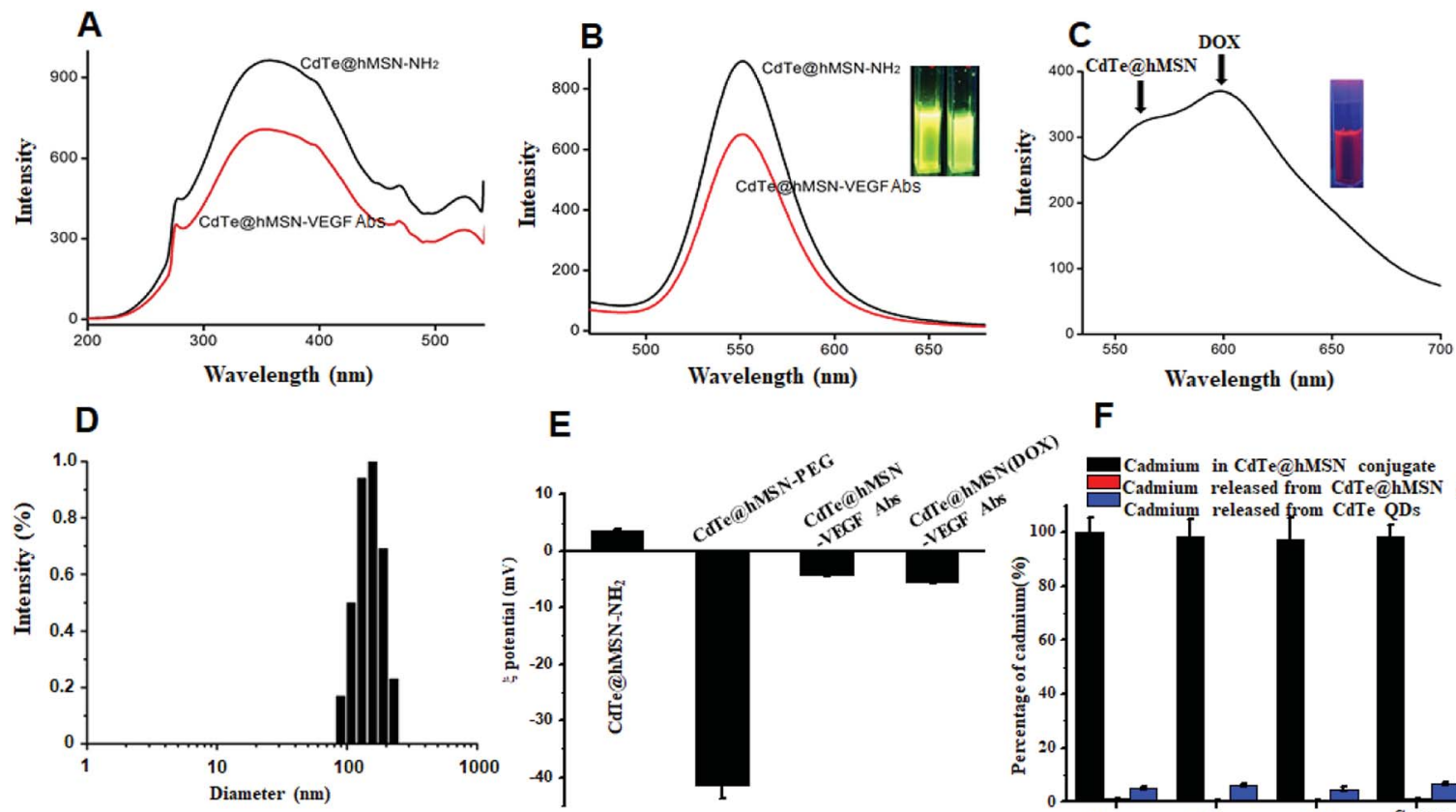

$\mathbf{F}$

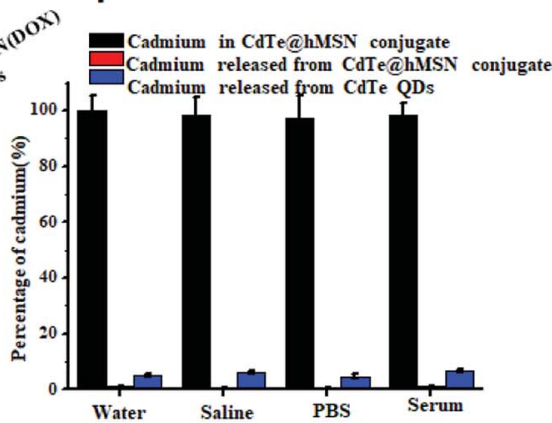

Fig. 2 Characteristics of CdTeahMSN conjugates. (A) Excitation spectra of CdTeahMSN conjugates; (B) emission spectra of CdTe(ahMSN conjugates; (C) emission spectra of CdTeahMSN(DOX)-VEGF Abs; (D) DLS curve of CdTeahMSN-VEGF Abs; (E) ऊ-potential of CdTe@ahMSN conjugates; (F) ICP-AES testing of cadmium release. 

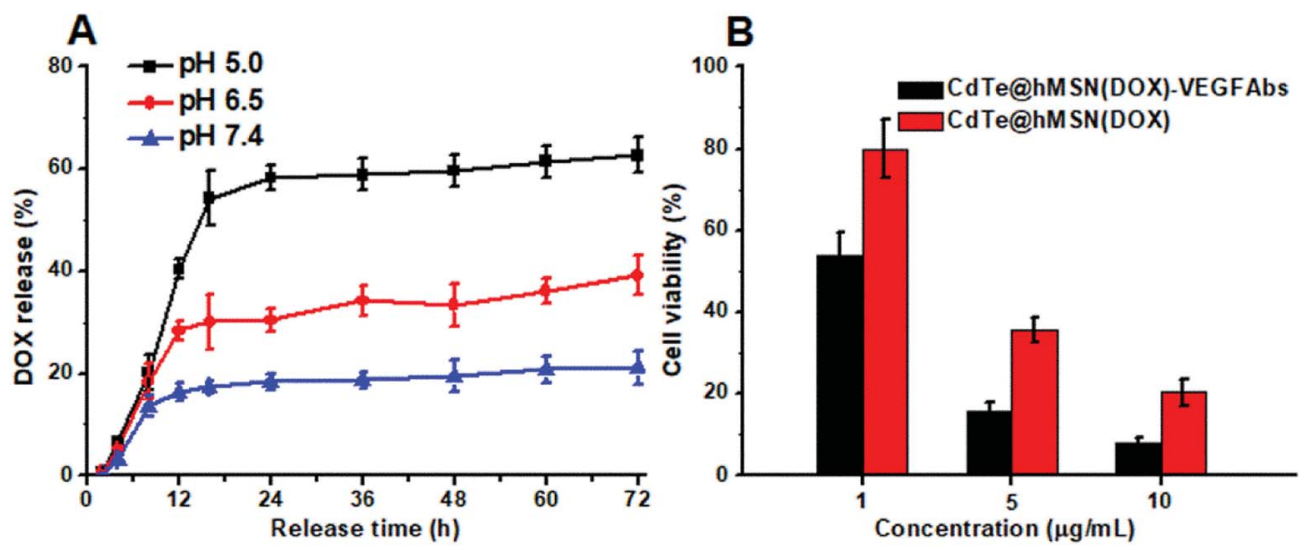

Fig. 3 DOX release curves in different media and cell viability test for released DOX. (A) DOX release curves in different media; (B) HeLa cell viability in the presence of CdTeahMSN conjugates at $\mathrm{pH} 6.5$.

confirmed that DOX can be loaded into CdTe@hMSN-PEG with high efficiency and the release behavior is $\mathrm{pH}$-dependent. Meanwhile, the effects of CdTe@hMSN(DOX) and CdTe@hMSN(DOX)-VEGF Abs on HeLa cell viability were also tested. Compared with the CdTe@hMSN(DOX) group, CdTe@hMSN(DOX)-VEGF Abs exhibited cytotoxicity (in Fig. 3B). With the addition of target VEGF Abs, the tumor cell death significantly increased, which further verified VEGF Abs specificity and enhanced DOX release.

\section{In vitro tumor cell targeting}

Two cell lines were used for in vitro evaluation of CdTe@hMSN conjugates: VEGF-positive HeLa cells and VEGF-negative L929 cells. Flow cytometry analysis and microscopy studies of CdTe@hMSN and CdTe@hMSN-VEGF Abs were conducted with CdTe QDs as the fluorophore (green color). DAPI staining (blue color) was used to identify the location of nucleus. As shown in the FASC data from Fig. 4B, incubation with $5 \mu \mathrm{g} \mathrm{mL}$

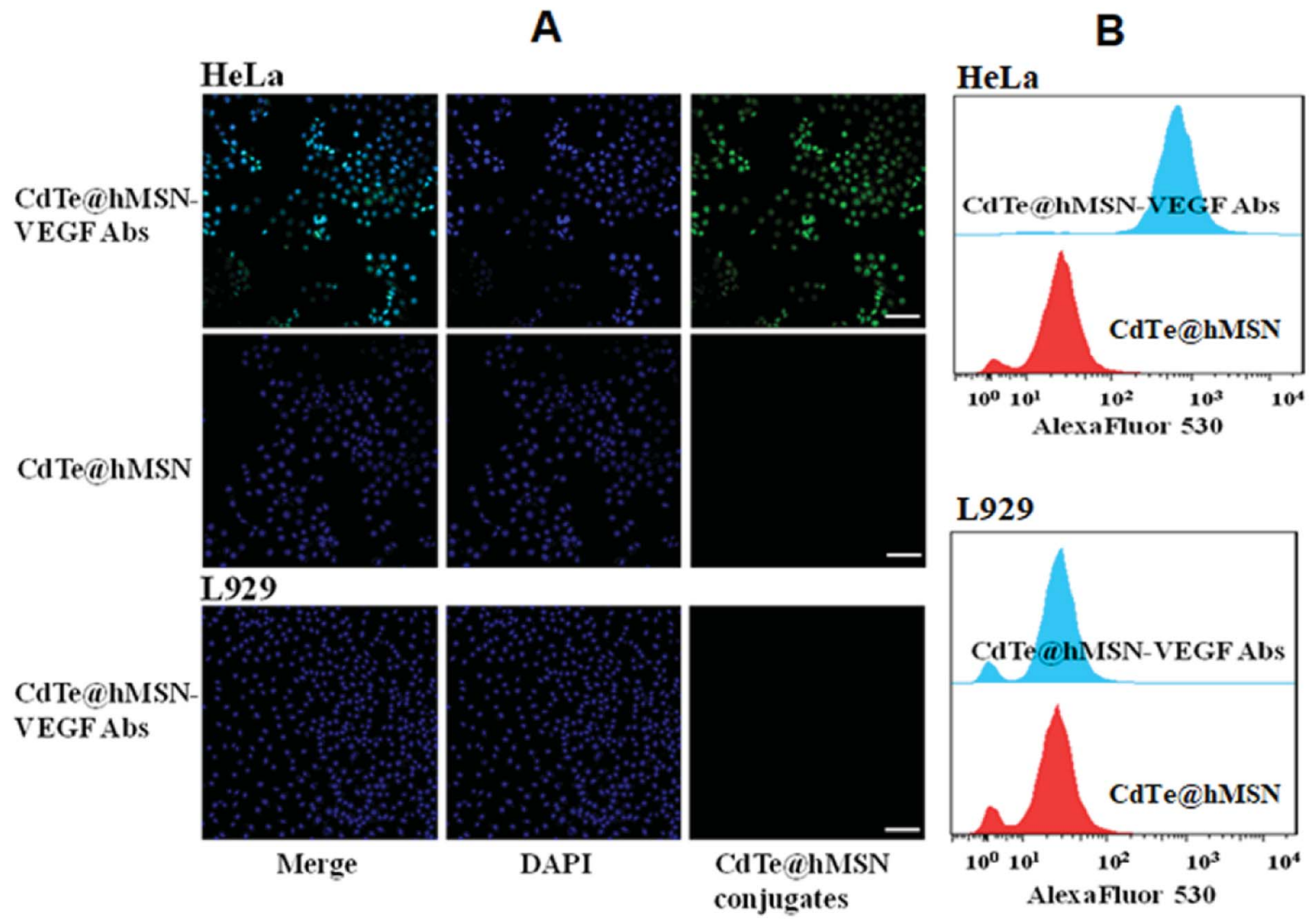

Fig. 4 In vitro assay of CdTeahMSN conjugates. (A) confocal fluorescence microscopy evaluation of CdTe@hMSN conjugates; (B) flow cytometry analysis of CdTeahMSN conjugates. 

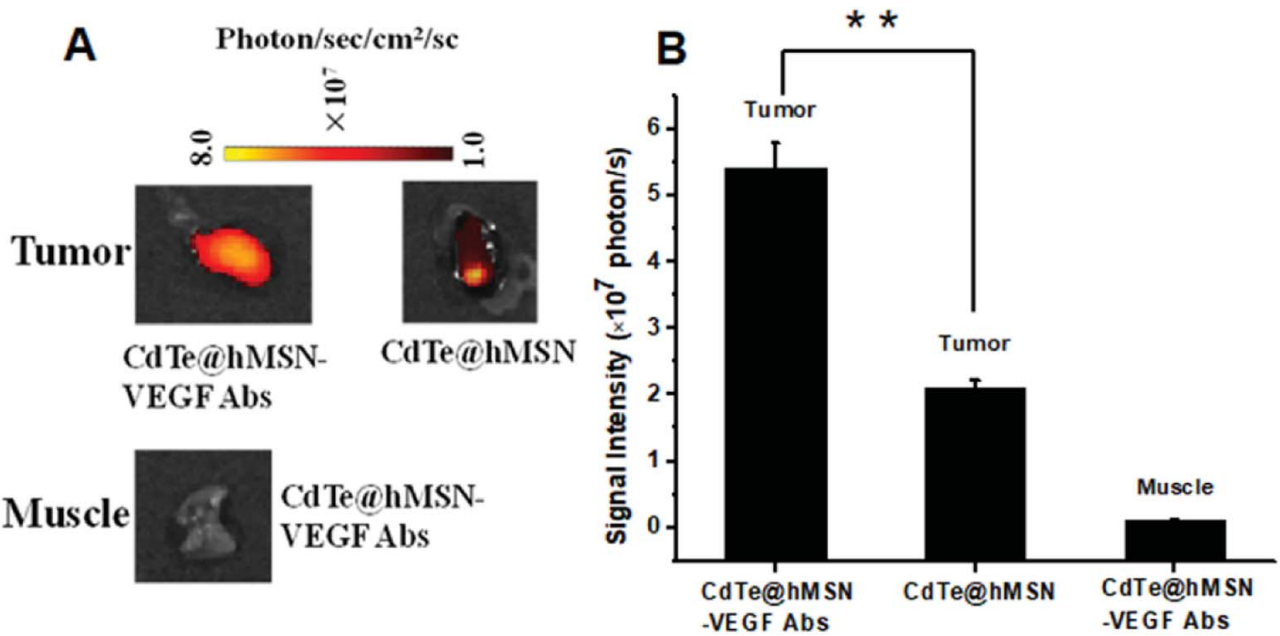

Fig. 5 DOX fluorescence imaging and quantification in tumors and muscles. (A) DOX fluorescence imaging in tumors and muscles; (B) DOX quantification in tumors and muscles.

CdTe@hMSN-VEGF Abs greatly enhanced the cellular fluorescence intensity compared with that from the CdTe@hMSN group at the same concentration $(\sim 20$ fold higher than that of the CdTe@hMSN group). On the contrary, CdTe@hMSN and
CdTe@hMSN-VEGF Abs exhibited very minimal specific binding with L929 cells. The VEGF Abs specificity was further validated by confocal fluorescence microscopy evaluation (Fig. 4A). In VEGF-positive HeLa cells, the fluorescence intensity

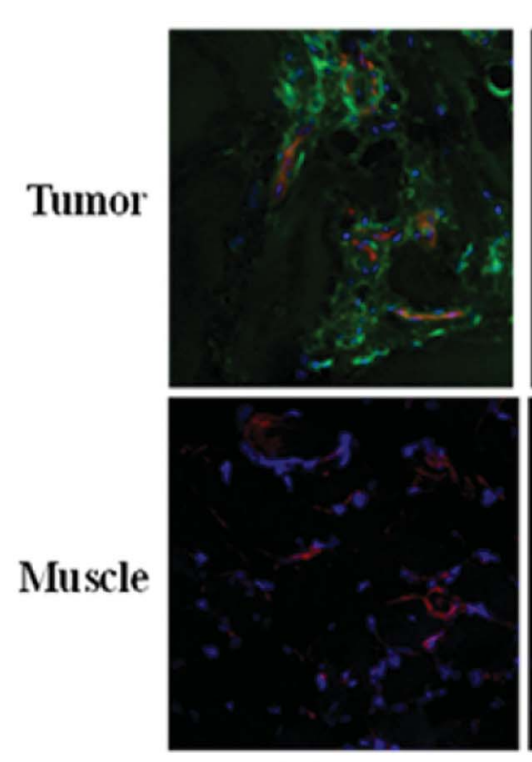

Merge

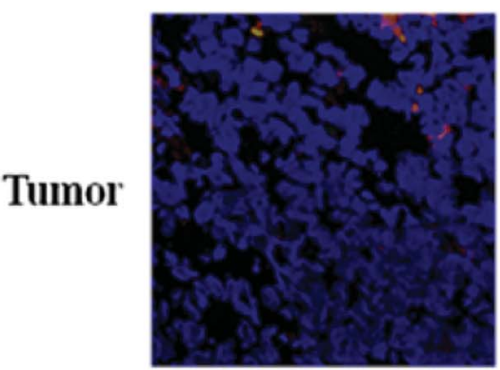

Merge
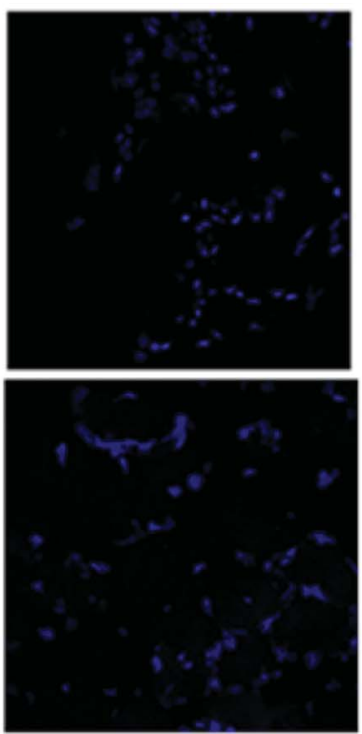

DAPI

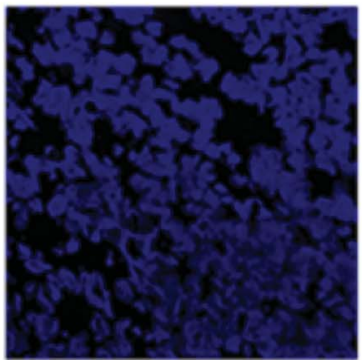

DAPI
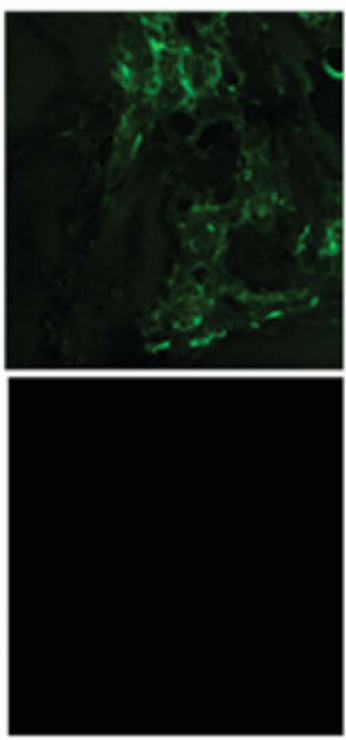

CdTe@hMSNVEGF Abs

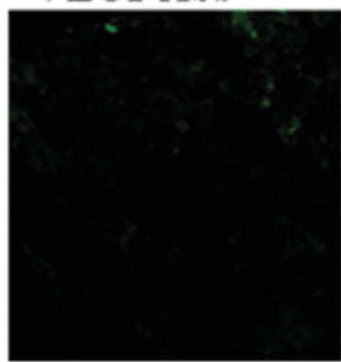

CdTe@hMSN
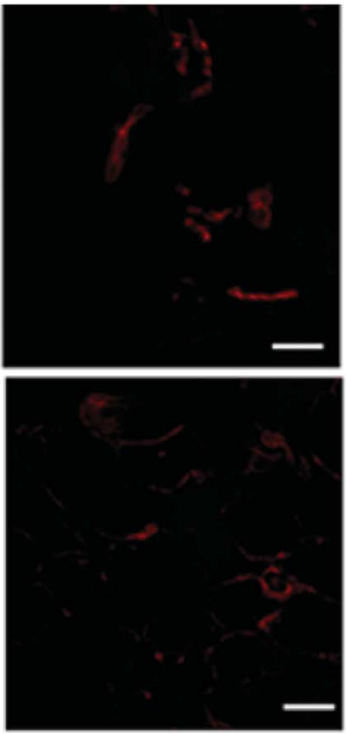

CD31

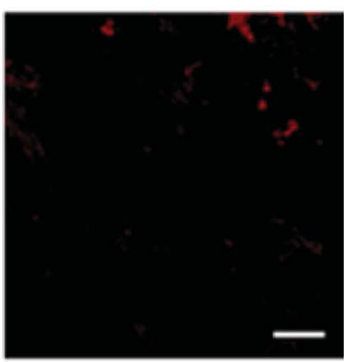

CD31

Fig. 6 Immunofluorescence staining of tumor nodules and muscles. 
from the CdTe@hMSN-VEGF Abs group was substantially stronger than that from the CdTe@hMSN group. The distribution pattern of DAPI overlapped well with that from CdTe, partly indicating that the nano-system entered the nuclei after cellular internalization. CdTe@hMSN-VEGF Abs did not show a clear cellular fluorescence signal in VEGF-negative L929 cells.

\section{Enhanced DOX delivery to tumors}

The feasibility of enhanced DOX delivery of CdTe@hMSN-VEGF Abs at tumor sites was studied by ex vivo fluorescence since DOX loaded in CdTe@hMSN conjugates exhibited strong fluorescence emission. After intravenous injection of CdTe@hMSN(DOX)-VEGF Abs or CdTe@hMSN(DOX) for $2 \mathrm{~h}$, tumors and muscles were collected and imaged on a NightOWL II system (ex/em $=485 / 600 \mathrm{~nm}$, where CdTe QDs have no effect on the fluorescence). As shown in Fig. 5A, a strong optical signal from DOX could be observed for CdTe@hMSN(DOX)-VEGF Abs group. Quantitative data from ROI analysis of the tumors and muscles (Fig. 5B) showed significantly higher fluorescent signal of DOX for CdTe@hMSN(DOX)-VEGF Abs, which was about 2.5fold that for CdTe@hMSN(DOX), clearly demonstrating the feasibility of delivering higher amounts of anticancer drugs to tumor sites in vivo using the VEGF antibody-conjugated CdTe@hMSN system.

\section{Histology}

To further confirm high accumulation of CdTe@hMSN conjugates in tumors, histological studies were carried out. Mice injected with CdTe@hMSN-VEGF Abs and CdTe@hMSN were euthanized at $2 \mathrm{~h}$ p.i. and then, HeLa tumor nodules and muscles were frozen and cryo-sectioned for immunofluorescence staining. Tumors treated with CdTe@hMSN-VEGF Abs and CdTe@hMSN were frozen for histological analysis. With negligible CdTe@hMSN conjugate uptake, the muscle tissue was also examined as a normal control. The green fluorescence in Fig. 6 is ascribed to the presence of CdTe@hMSN conjugates as CdTe exhibits emission at $550 \mathrm{~nm}$. Red fluorescence was from CD31, a vasculature marker. From the green fluorescence intensity and distribution, a substantial amount of CdTe@hMSN-VEGF Abs was accumulated in HeLa tumors, which was significantly higher than that for the CdTe@hMSN group. CdTe@hMSN-VEGF Abs was primarily located on the tumor vasculature, which indicated that tumor vasculaturetargeting is truly responsible for the enhanced tumor uptake of CdTe@hMSN conjugates. However, the partial overlap of green and red fluorescence (which delineates CD31) indicated some extravasation of CdTe@hMSN conjugates from vasculature at that time. Overlap of green and blue fluorescence verified that CdTe@hMSN conjugates could enter the nuclei, which was consistent with the results from cell staining. No observable green fluorescence was detected in muscles.

\section{Conclusion}

In conclusion, a core/shell CdTe@hMSN-VEGF Abs system was designed and synthesized for enhanced drug delivery and accumulation in tumors. The optical properties of CdTe QDs from CdTe@hMSN conjugates remain stable due to the protection of hMSN, which could be used for immunostaining directly without further modifying with fluorescence dye. Relatively high amount of DOX could be loaded in CdTe@hMSN conjugates, and $\mathrm{pH}$-dependent DOX release behavior was detected. Relying on the VEGF target, the accumulation of CdTe@hMSN conjugates and enhanced DOX delivery to HeLa tumors in vivo were demonstrated in tumor-bearing mice. A problem that cannot be ignored in this study is that CdTe QDs are not optimal materials due to their potential toxicity and limited fluorescence emission wavelength. This has encouraged us to develop biomedical agents with higher biocompatibility and longer emission wavelength in the future study.

\section{Conflicts of interest}

There are no conflicts to declare.

\section{Acknowledgements}

This work is supported by Natural Science Foundation of Jiangsu Province, China (BK20161173) and the Program for Distinguished Talents of Six Domains in Jiangsu Province, China (2014-SWYY-007), Postgraduate Research \& Practice Innovation Program of Jiangsu Province, China (KYCX18_2200) and Xuzhou Natural Science Foundation, China (KC18201 and KC18108).

\section{References}

1 C. Yang, M. Zhang and D. Merlin, J. Mater. Chem. B, 2018, 6, 1312-1321.

2 J. Liu, J. Dong, T. Zhang and Q. Peng, J. Controlled Release, 2018, 286, 64-73.

3 E. Niezabitowska, J. Smith, M. R. Prestly, R. Akhtar, F. W. von Aulock, Y. Lavallée, H. Ali-Boucetta and T. O. McDonald, RSC Adv., 2018, 8, 16444-16454.

4 M. R. Chao, C. W. Hu and J. L. Chen, Biosens. Bioelectron., 2014, 61, 471-477.

5 Y. Du, D. Z. Yang, S. A. Sun, Z. M. Zhao and D. Q. Tang, Luminescence, 2015, 30, 519-525.

6 A. Zavari-Nematabad, M. Alizadeh-Ghodsi, H. Hamishehkar, E. Alipour, Y. Pilehvar-Soltanahmadi and N. Zarghami, Anal. Bioanal. Chem., 2017, 409, 1301-1310.

7 J. Y. Wang, J. F. Zhao, P. N. Wang, W. L. Yang and J. Y. Chen, J. Fluoresc., 2011, 21, 1635-1642.

8 Y. Li, Y. Zhou, X. Li, J. Sun, Z. Ren, W. Wen, X. Yang and G. Han, RSC Adv., 2016, 6, 38365-38370.

9 W. Ngamcherdtrakul, T. Sangvanich, M. Reda, S. Gu, D. Bejan and W. Yantasee, Int. J. Nanomed., 2018, 13, 40154027.

10 Q. Yue, J. L. Li, Y. Zhang, X. W. Cheng, X. Chen, P. P. Pan, J. C. Su, A. A. Elzatahry, A. Alghamdi, Y. H. Deng and D. Y. Zhao, J. Am. Chem. Soc., 2017, 139, 15486-15493.

11 Y. Zhao, L. N. Lin, Y. Lu, S. F. Chen, L. Dong and S. H. Yu, Adv. Mater., 2010, 22, 5255-5259. 
12 X. Jiang, T. L. Ward, Y. S. Cheng, J. Liu and C. J. Brinker, Chem. Commun., 2010, 46, 3019-3021.

13 D. Z. Yang, X. Y. Yao, J. J. Dong, N. Wang, Y. Du, S. Sun, L. P. Gao, Y. Y. Zhong, C. T. Qian and H. Hong, Bioconjugate Chem., 2018, 29, 2776-2785.

14 T. Li, G. Kang, T. Wang and H. Huang, Oncol. Lett., 2018, 16, 687-702.

15 A. Bahreyni, M. Khazaei, M. Rajabian, M. Ryzhikov, A. Avan and S. M. Hassanian, J. Pharm. Pharmacol., 2018, 70, 191196.

16 B. Du, W. Liu, Y. Deng, S. Li, X. Liu, Y. Gao and L. Zhou, Int. J. Nanomed., 2015, 10, 2555-2565.

17 L. Li, S. Pan, B. Ni and Y. Lin, Cell Biol. Int., 2014, 38, 962970.

18 S. Huang, K. Shao, Y. Liu, Y. Kuang, J. Li, S. An, Y. Gao, H. Ma and C. Jiang, ACS Nano, 2013, 7, 2860-2871.

19 J. S. Rader, M. W. Sill, J. H. Beumer, H. A. Lankes, D. M. Benbrook, F. Garcia, C. Trimble, T. J. Tate, R. Lieberman, R. E. Zuna, C. A. 3rd Leath, N. M. Spirtos,
J. Byron, P. H. Thaker, S. Lele and D. Alberts, Gynecol. Oncol., 2017, 145, 291-297.

20 X. Zhu, K. Er, C. Mao, Q. Yan, H. Xu, Y. Zhang, J. Zhu, F. Cui, W. Zhao and H. Shi, Cell. Physiol. Biochem., 2013, 32, 64-73. 21 K. S. Siveen, K. Prabhu, R. Krishnankutty, S. Kuttikrishnan, M. Tsakou, F. Q. Alali, S. Dermime, R. M. Mohammad and S. Uddin, Curr. Vasc. Pharmacol., 2017, 15, 339-351.

22 L. Li, H. Qian and J. Ren, J. Lumin., 2006, 116, 59-66.

23 Z. Deng, Y. Zhang and Q. Wei, J. Phys. Chem. B, 2007, 111, 12024-12031.

24 Y. Chen, H. R. Chen, L. M. Guo, Q. J. He, F. Chen, J. Zhou, J. Feng and J. Shi, ACS Nano, 2010, 4, 529-539.

25 X. Fang, C. Chen, Z. Liu, P. Liu and N. Zheng, Nanoscale, 2011, 3, 1632-1639.

26 F. Chen, H. Hong, S. Shi, S. Goel, H. F. Valdovinos, R. Hernandez, C. P. Theuer, T. E. Barnhart and W. Cai, Sci. Rep., 2014, 4, 5080-5090.

27 National Research Council, Guide for the Care and Use of Laboratory Animals, The National Academies Press, Washington, DC, 8th edn, 2011. 